\title{
Promoting Youth Self-Regulation through Psychotherapy: Redesigning Treatments to Fit Complex Youth in Clinical Care
}

\section{Citation}

Weisz, John R. 2015. Promoting Youth Self-Regulation through Psychotherapy: Redesigning Treatments to Fit Complex Youths in Clinical Care. In Self-Regulation in Adolescence, eds. Gabriele Oettingen and Peter M. Gollwitzer: 311-332.

\section{Published Version}

doi:10.1017/CB09781139565790.016

\section{Permanent link}

http://nrs.harvard.edu/urn-3:HUL.InstRepos:30778756

\section{Terms of Use}

This article was downloaded from Harvard University's DASH repository, and is made available under the terms and conditions applicable to Other Posted Material, as set forth at http:// nrs.harvard.edu/urn-3:HUL.InstRepos:dash.current.terms-of-use\#LAA

\section{Share Your Story}

The Harvard community has made this article openly available.

Please share how this access benefits you. Submit a story.

\section{Accessibility}




\title{
15 Promoting Youth Self-Regulation through Psychotherapy: Redesigning Treatments to Fit Complex Youths in Clinical Care
}

\author{
John R. Weisz
}

\section{Author Note}

John R. Weisz, Department of Psychology, Harvard University

Some of the research reported here was supported by grants from the National Institute of Mental Health (MH57347, MH068806, MH085963), the Norlien Foundation, the MacArthur Foundation, and the Annie E. Casey Foundation. I am grateful to an array of wise colleagues and students, and to the youths, families, clinicians, clinic administrators, and government program and policy leaders who have participated in and supported our research and enriched our thinking.

Correspondence concerning this chapter should be addressed to John R. Weisz, Department of Psychology, Harvard University, William James Hall, 33 Kirkland Street, Cambridge, Massachusetts 02138,or E-mail john_weisz@harvard.edu.

\section{Abstract}

Improving self-regulation can be seen as a central objective of youth psychotherapy. Five decades of psychotherapy research have produced an array of evidence-based psychotherapies (EBPs), but meta-analyses have shown that these EBPs show only a modest advantage over treatments-as-usual. This suggests a need for therapies that can clearly improve upon usual practice. The Modular Approach to Therapy for Children protocol (MATCH; Chorpita \& Weisz, 2009) was designed to meet this objective. MATCH integrates treatment components involving at least three forms of self-regulation and addressing anxiety, depression, and misconduct. An initial randomized trial of MATCH showed significant support, in comparison to both usual care and standard EBPs. Further improvements in MATCH and other treatments may come about through increased focus on strengthening selfregulation in cognitive, emotional, and behavioral forms.

In the very full agenda of what girls and boys need to accomplish during development, a centerpiece is the shift from external monitoring and control 
to self-regulation. Psychotherapy with children and adolescents (herein "youth") has diverse goals, but many of these fit within the broad rubric of promoting effective self-regulation. This chapter focuses on what has been learned from youth psychotherapy research, what self-regulation processes appear to be involved, an emerging strategy for improving therapy outcomes for clinically referred youths, and ways an improved understanding of selfregulation may enhance and strengthen youth psychotherapy.

\section{Historical Background: The Evolution of Youth Psychotherapy}

Some of the core processes involved in psychotherapy can be traced back to the classical Greek era, when human discourse was used as a means of exploring the contents of the mind. For example, Socrates' (469-399 BCE) teaching approach, later dubbed the "Socratic method," involved questioning others to prompt them to examine their beliefs, and this approach is reflected in many modern therapies. The birth of psychotherapy proper is often linked to psychoanalysis and the work of Sigmund Freud (1856-1939), but diverse other models have had major impact, including psychodynamic, behavioral, and cognitive.

Understandably, the practice of youth psychotherapy has grown much faster than research on its effects. In fact, early findings of the research that was done were discouraging, suggesting that therapy might be no more effective than the mere passage of time (Levitt, 1957, 1963). However, over time, some therapies began to be clearly documented via protocols or "manuals," research methods grew more rigorous, and findings grew more encouraging.

\section{Evaluating Youth Psychotherapy: Meta-Analytic Findings}

Experimental designs for testing therapies are now diverse, but the randomized controlled trial (RCT) is the most widely accepted of these designs. We emphasize the RCT evidence in this chapter, with findings summarized via the effect size (ES) statistic, an index of the magnitude and direction of treatment impact. The ES is the post-treatment difference, on a particular outcome measure, between the treatment and comparison groups, divided by the standard deviation of the measure. We calculate ES such that positive values reflect an advantage of the target treatment group over the comparison or control group. Cohen (1988) has suggested cutoffs of .20, .50, and .80 as benchmarks for small, medium, and large effects, respectively. 


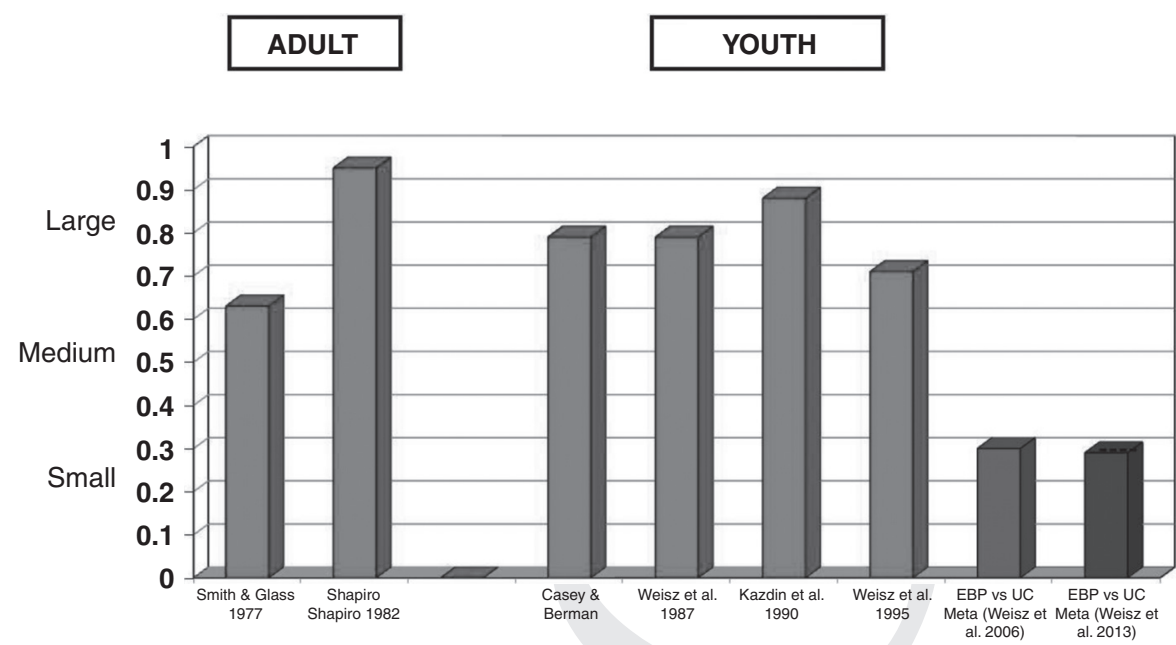

Figure 15.1. Mean effect sizes found in two broad-based meta-analyses of adult psychotherapy effects (Shapiro \& Shapiro, 1982; Smith \& Glass, 1977), four broad-based meta-analyses of youth psychotherapy effects (Casey \& Berman, 1985; Kazdin et al., 1990; Weisz et al., 1987; Weisz et al., 1995), and two meta-analyses of randomized trials comparing evidence-based youth psychotherapies to usual care (Weisz, Jensen-Doss, \& Hawley, 2006; Weisz, Kuppens, Eckshtain, Ugueto, Hawley, \& Jensen-Doss, 2013). [Published with permission, John R. Weisz]

ES values can be synthesized across multiple studies, and across multiple outcome measures within each study, in a process called meta-analysis. Meta-analyses have helped build a cumulative understanding of youth psychotherapy effects over the past 25 years. Four meta-analyses of youth psychotherapy studies (shown in Figure 15.1) are particularly broad in their inclusion criteria, encompassing a range of treated problems and treatment methods. In the earliest of these broad-based meta-analyses, Casey and Berman (1985) included outcome studies involving children aged 12 and younger, and found a mean ES of .71. Weisz, Weiss, Alicke, and Klotz (1987) included both child and adolescent studies, and found a mean ES of .79. In a third meta-analysis, Kazdin, Bass, Ayers, and Rodgers (1990) included ages 4-18, and found a mean ES of .88. Finally, Weisz, Weiss, Han, Granger, and Morton (1995) included ages 2-18, and found a mean ES of .71. These four meta-analyses reported relatively similar effect sizes near Cohen's (1988) 'large' effect threshold. These youth therapy effects fall roughly within the range of adult psychotherapy effects (see two bars at the left in Figure 15.1: Shapiro \& Shapiro, 1982; Smith \& Glass, 1977). Note, however, that when 
weighted for sample size and heterogeneity of variance, the youth therapy ES means are actually closer to 'medium' than 'large' (Weisz et al., 1995).

\section{Evidence-Based Psychotherapies (EBPs) and Their Relation to Self-Regulation}

The growth of the youth psychotherapy evidence base has prompted efforts to identify the specific therapies sufficiently well supported to be considered "empirically validated" or "evidence-based." Task forces and review teams have distilled outcome evidence and grouped therapies according to their level of empirical support (see, e.g., Silverman \& Hinshaw, 2008). Treatments that have substantial empirical support (e.g., multiple supportive RCTs) are now often referred to as evidence-based psychotherapies (EBPs), and there are currently dozens of youth EBPs, particularly for depression, anxiety, and conduct-related problems (reviewed in Weisz, 2004).

A related development has been the search for "mechanisms of change" that is, change processes evident during treatment that can explain why the treatment works when it does. A number of mechanisms have been proposed for various treatments, and some evidence shows that some of these function as statistical mediators in relation to treatment outcome (see e.g., Weisz, Ng, Rutt, Lau, \& Masland, 2013). However, there is still little robust, replicated evidence identifying specific change mechanisms. It is possible that the search for mechanisms could be informed and enriched by a focus on forms of youth self-regulation that seem to underlie the goals of so many youth therapies. Although the EBPs are not usually discussed in this way, I believe that at least three forms of self-regulation are targeted in these treatments.

\section{Cognitive Regulation}

Cognitive regulation is used in various ways within EBPs for multiple youth problems, but it is a centerpiece of the most thoroughly studied EBP for youth depression - cognitive-behavioral therapy (CBT). In CBT for youth depression, cognitive regulation takes such forms as teaching the young client how thoughts can lead to feelings, how thoughts may or may not be accurate ("Just because you think it doesn't mean it's true."), how unrealistically negative thoughts can lead to sad feelings, and how those unrealistic thoughts may be reevaluated and modified to make them more realistic and thus reduce the symptoms of depression. Another example of cognitive regulation in CBT for youth depression is teaching youngsters strategies for 
systematic problem solving when they face stressors and dilemmas; these are active strategies used to combat the passive, helpless responses that are common among depressed youths. In our youth depression CBT program, for example, we teach youngsters to use the STEPS procedure when they face a problem: Say what the problem is, Think of possible solutions, Examine each one for its pros and cons, Pick one, and See if it works when you try it; if not, try one of your other solutions. Youth treatment using CBT for depression often involves multiple sessions of practice with the therapist in the use of such cognitive regulation strategies, complemented by weekly homework assignments in which youngsters practice the skills in their everyday lives. A goal of these experiences is to turn the skills inward in the form of cognitive self-regulation.

\section{Emotion Regulation}

Emotion regulation is involved in multiple EBPs for multiple youth problems, but it is especially central to the most thoroughly tested treatment approach for youth anxiety: exposure. Exposure is typically graduated, with the youngster progressing from facing low-level fears to ever-scarier fears, until s/he can do what is needed for successful adaptation. Consider, for example, a girl who fears separation from her mother, still sleeps in her mother's bed, and often misses school because separating from her mother is too scary. The therapist using exposure will first work with the girl to create a fear hierarchy, listing and ranking the scariness of, say, a dozen situations involving separation from Mom; a low level might be eating alone in the kitchen with Mom upstairs, a middle level might be shopping in different stores in the same mall, and a high level might be a full day at school apart from Mom. Graduated exposures would involve mastering fears low in the hierarchy, and working upward to higher levels. A key mechanism thought to underlie anxiety reduction in exposure is habituation: over time during an exposure, the level of fearful arousal may rise initially but eventually will begin to drop. Ideally, as boys and girls learn that sustained exposure leads to reduced anxiety, they come to tolerate and even initiate exposures, doing the things they need to do for successful functioning at home, in school, and with peers. In other words, a critical goal of exposure treatment is to guide the youth toward this particular form of self-regulation of emotion.

\section{Behavioral Regulation}

While effective behavioral regulation is a central objective of most youth EBPs, it is particularly prominent in treatments for youth conduct problems. The best-documented treatment successes in this domain, particularly for 
children and early adolescents, involve teaching caregivers skills to maximize their sons' and daughters' regulation of behavior. Caregivers are taught, for example, how to give instructions that are clear and crisp - and thus most likely to generate an obedient response - and how to focus their attention and praise on the youngster's positive behavior - for example, following family rules, and addressing frustrations through discussion rather than tantrums. The holy grail of behavioral parent training, and in fact of most behavioral interventions, is generalization - that is, appropriate behavior that is sustained even when parents and others are not there to provide the perfect environmental response. Another way to think about such generalization is that it reflects a shift toward internal management, self-regulation, of behavior.

\section{Summary: Evidence-Based Psychotherapies and Self-Regulation}

Evidence that has accumulated over five decades, synthesized through metaanalyses, has been used to identify youth EBPs, psychotherapies supported in multiple studies. Researchers are trying to understand how these EBPs work by identifying the mechanisms of change through which treatment benefits come about. The search for these mechanisms may be informed by focusing on three forms of self-regulation that appear central to youth EBPs: cognitive, emotional, and behavioral.

\section{Concerns about the Evidence-Based Psychotherapies}

The EBPs that appear so relevant to cognitive, emotional, and behavioral regulation have been identified as evidence-based because of empirical evidence supporting their efficacy. However, several concerns have arisen about the nature and strength of these EBPs and their supportive evidence (see Weisz \& Gray, 2008).

\section{Most EBPs Have Not Been Tested in the Context of Actual Clinical Practice}

The conditions in which most EBPs have been developed and tested tend to differ markedly from the conditions of real-world clinical practice. This limits our ability to generalize from the research findings. Youths referred to clinical care settings tend to differ from those recruited for efficacy trials in diverse ways, including severity and family adversity (Hammen, Rudolph, Weisz, Burge, \& Rao, 1999; Southam-Gerow, Weisz, \& Kendall, 2003). Therapists who work in clinics tend to differ in professional goals, work pressures, and numerous other ways from therapists employed as 
research clinicians in clinical trials (Weisz \& Addis, 2006). For example, therapists who work in clinics typically treat a broad array of problems and disorders, often within the same day, and this limits the value to them of developing narrow expertise in a single treatment for a single disorder. Clinical practice and research settings also tend to differ in a broad range of ways that could affect treatment outcomes. In clinical practice settings, for example, a variety of time, productivity, financial, and other work pressures can limit therapists' ability to learn EBPs, their time to receive supervision in the use of those interventions with the youths they are treating, and even their time to do the kind of assessment and treatment planning that are often critical to the successful use of EBPs (Weisz \& Addis, 2006). Such differences between the youths, therapists, and settings of research and those of everyday clinical care highlight the need to test EBPs under conditions that match those of clinical practice - to determine how well the EBPs might fare when thrust into the crucible of real life.

We have found (Weisz, Jensen-Doss, \& Hawley, 2005) that the research literature on EBPs does not provide a very clear picture of how these treatments fare under real-world practice conditions. Most studies of youth psychotherapy effects - reviewed for that 2005 article - were conducted in settings constructed for research (e.g., university laboratories or study rooms in a school, not in mental health clinics), with treatment provided to youths who were recruited for the study (not clinically referred), and with treatment provided by graduate students or research employees (not clinical practitioners). Table 15.1 shows that across all the studies, only $13 \%$ of the study samples were clinically referred, treatment-seeking youth; only $19 \%$ of the studies employed even one practicing clinician as a treatment provider; and in only $4 \%$ of the studies was treatment provided in an actual clinical service setting separate from the research program. Summing across dimensions, as shown in Table 15.1, we found that only $1 \%$ of the studies reviewed included any clinically referred youths, at least one practicing clinician, and some treatment carried out in a clinical service setting. Thus, the research provided little information about how the tested youth psychotherapies would fare in real-world clinical care conditions.

\section{Most EBPs Have Not Been Tested in Comparison to Usual Clinical Care}

The youth psychotherapy literature also provides relatively little information on a related question: Do EBPs produce better outcomes than the treatments youths receive in usual clinical care? In some respects, this is the most basic 
Table 15.1. Clinical (un)Representativeness of the Youth Treatment Evidence Base: Youths, Therapists, and Treatment Settings Employed in Outcome Studies

\begin{tabular}{|c|c|c|c|c|c|}
\hline & Anxiety & Depression & ADHD & Conduct & $\begin{array}{l}\text { All } \\
\text { Studies }\end{array}$ \\
\hline \multicolumn{6}{|l|}{$\begin{array}{l}\text { How youths enrolled in } \\
\text { the study: }\end{array}$} \\
\hline $\begin{array}{l}\% \text { Recruited, not } \\
\text { treatment-seeking }\end{array}$ & 90.24 & 77.78 & 87.50 & 60.42 & 76.69 \\
\hline $\begin{array}{l}\text { \% Treatment-seeking, } \\
\text { clinic-referred }\end{array}$ & 3.66 & 16.67 & 12.50 & 19.79 & 12.71 \\
\hline $\begin{array}{l}\text { \% Required via } \\
\text { court/justice system }\end{array}$ & 1.22 & 0.00 & 0.00 & 17.71 & 7.63 \\
\hline$\%$ Studies not reporting & 4.88 & 5.56 & 0.00 & 2.08 & 2.97 \\
\hline \multicolumn{6}{|c|}{$\begin{array}{l}\text { Who provided the treatment: } \\
\text { \% With any }\end{array}$} \\
\hline $\begin{array}{l}\text { researchers/grads } \\
\text { \% With any }\end{array}$ & 57.32 & 47.06 & 45.00 & 38.54 & 47.21 \\
\hline paraprofessionals & 20.73 & 11.11 & 12.50 & 22.92 & 19.49 \\
\hline $\begin{array}{l}\% \text { With any practicing } \\
\text { clinicians }\end{array}$ & 1.22 & 55.56 & 10.00 & 30.21 & 18.64 \\
\hline$\%$ Studies not reporting & 28.05 & 11.11 & 40.00 & 19.79 & 25.42 \\
\hline \multicolumn{6}{|l|}{$\begin{array}{l}\text { Setting where treatment } \\
\text { took place: }\end{array}$} \\
\hline \% Research settings & 50.00 & 44.44 & 42.50 & 48.96 & 47.88 \\
\hline $\begin{array}{l}\% \text { Clinical service } \\
\text { settings }\end{array}$ & 2.44 & 5.56 & 0.00 & 7.29 & 4.24 \\
\hline$\%$ Correctional settings & 1.22 & 0.00 & 0.00 & 7.29 & 3.39 \\
\hline$\%$ Studies not reporting & 46.34 & 50.00 & 55.00 & 37.50 & 44.49 \\
\hline $\begin{array}{l}\text { Representativeness sum: } \\
\text { \% Reporting no rep } \\
\text { factors }\end{array}$ & 92.68 & 38.89 & 77.50 & 55.21 & 70.76 \\
\hline $\begin{array}{l}\text { \% Reporting one rep } \\
\text { factor }\end{array}$ & 7.32 & 50.00 & 22.50 & 34.38 & 24.15 \\
\hline $\begin{array}{l}\% \text { Reporting two rep } \\
\text { factors } \\
\% \text { Reporting all three }\end{array}$ & 0.00 & 5.56 & 0.00 & 8.33 & 3.81 \\
\hline rep factors & 0.00 & 5.56 & 0.00 & 2.08 & 1.27 \\
\hline
\end{tabular}

(Published with permission, John R. Weisz)

question many in clinical practice might ask. Building competence in a typical manual-guided treatment protocol requires a major investment of time and funds, and is especially challenging for clinicians who are managing the time demands of a practice career. Thus, it seems fair for practitioners to ask whether learning a particular EBP will lead to better clinical outcomes than current practices do. 


\section{In Studies Comparing EBPs to Usual Care, the Findings Raise Important Questions}

EBP proponents have suggested that EBPs should be used instead of usual clinical care; others have raised concerns about EBPs and their manuals. Various writers have suggested that EBPs (a) may not work well with the complex and severe cases often seen in usual clinical care; (b) may not fare so well when they confront co-occurring problems and comorbid diagnoses, or shifts during treatment in the most pressing problems, both of which are common in usual clinical care; and (c) are so rigidly structured that they make it hard to individualize treatment to fit client needs. The concerns reflect the view that many manual-guided EBPs may not fare so well with clinically referred individuals in usual clinical care (Addis \& Krasnow, 2000; Havik \& Van den Bos, 1996; Westen, Novotny, \& Thompson-Brenner, 2004).

We have conducted two meta-analyses exploring the performance of EBPs compared to usual care. The first (Weisz, Jensen-Doss, \& Hawley, 2006) included 32 randomized trials, spanning 1973-2004, each comparing EBPs to usual care. The second (Weisz et al., 2013) included 52 studies, spanning 1973-2010. In both meta-analyses, the EBPs outperformed usual care, but the advantage was modest. The mean effect sizes at post-treatment were .30 and .29 for the 2006 and 2013 meta-analyses, respectively. Both effects translate into a "common language effect size" of 58\% (McGraw \& Wong, 1992), meaning the probability was only .58 that a randomly selected youth receiving an EBP would be better off after treatment than a randomly selected youth receiving usual care. Revisiting Figure 15.1 helps us put the findings into context: the two bars at the left show the mean ES values found in meta-analyses of adult psychotherapy research; the next four bars show mean ES values from meta-analyses of youth psychotherapy research; and the two bars at the right show mean ESs in our two EBP vs. usual care meta-analyses; the drop-off in effects is quite clear.

\section{Summary: Concerns about Evidence-Based Psychotherapies in Relation to Everyday Treatment}

The EBPs, while promising, have certain limitations. Most of them have not been tested in conditions resembling real-world clinical care, so relatively little information is available on how well the treatments work with referred youths treated by clinical practitioners in actual clinical practice. The fact that most youth treatment research has been done in rather pristine experimental contexts may also have limited our understanding of how to fit the treatments into real-world care and make them effective in that context. 
This concern is underscored by two meta-analyses suggesting that EBPs are only modestly more effective than usual clinical care.

\section{Redesigning Evidence-Based Psychotherapies for Real-World Treatment}

Our EBP vs. usual care meta-analyses showed only a very modest advantageslightly above chance expectancy - of standard EBPs over the usual treatments young people receive. This suggests a need to improve the potency of EBPs with youths in everyday clinical service contexts. This has been one of the core objectives of our Research Network on Youth Mental Health (e.g., Schoenwald, Kelleher, \& Weisz, 2008). We have tried to address some of the limitations of EBPs identified by critics, particularly when those concerns matched our own, arising from our attempts to implement and test EBPs in such everyday treatment settings as community mental health clinics (e.g., Southam-Gerow et al., 2010; Weisz et al., 2009).

A number of these limitations derive from the fact that most EBPs are designed for a single problem or disorder (or a small homogeneous clustere.g., "defiant children," depressive disorders only). Learning a new treatment for a single disorder may not be so helpful to the average clinician, whose caseload is likely to be quite diverse diagnostically; that single disorder may represent only a tiny percentage of the youths seen in a typical week. A single-disorder focus also clashes with the fact that most clinically referred youths present with multiple, diverse problems and disorders (Angold, Costello, \& Erkanli, 1999; Jensen \& Weisz, 2002). Single disorder treatments also struggle when the initial focus of treatment changes midway through a treatment episode (e.g., when a teen being treated for depression becomes aggressive and is threatened with school expulsion); such situations call for flexibility and a capacity to shift focus during treatment - an option not available within single disorder treatments.

To address these challenges - that is, heterogeneous clinician caseloads, comorbidity, and flux in treatment needs - we redesigned treatment to accommodate an array of intervention targets, including problems and disorders involving anxiety, depression, and misconduct. Relying on surveys of the most common elements of EBPs within those three domains (e.g., Chorpita \& Dalaiden, 2009; Chorpita, Dalaiden, \& Weisz, 2005), and meta-analytic findings on effective treatments (e.g., Weisz et al., 1995), we assembled a treatment manual that integrated common elements of CBT for anxiety, CBT for depression, and behavioral parent training for conductrelated problems and disorders. The resulting treatment manual is called 


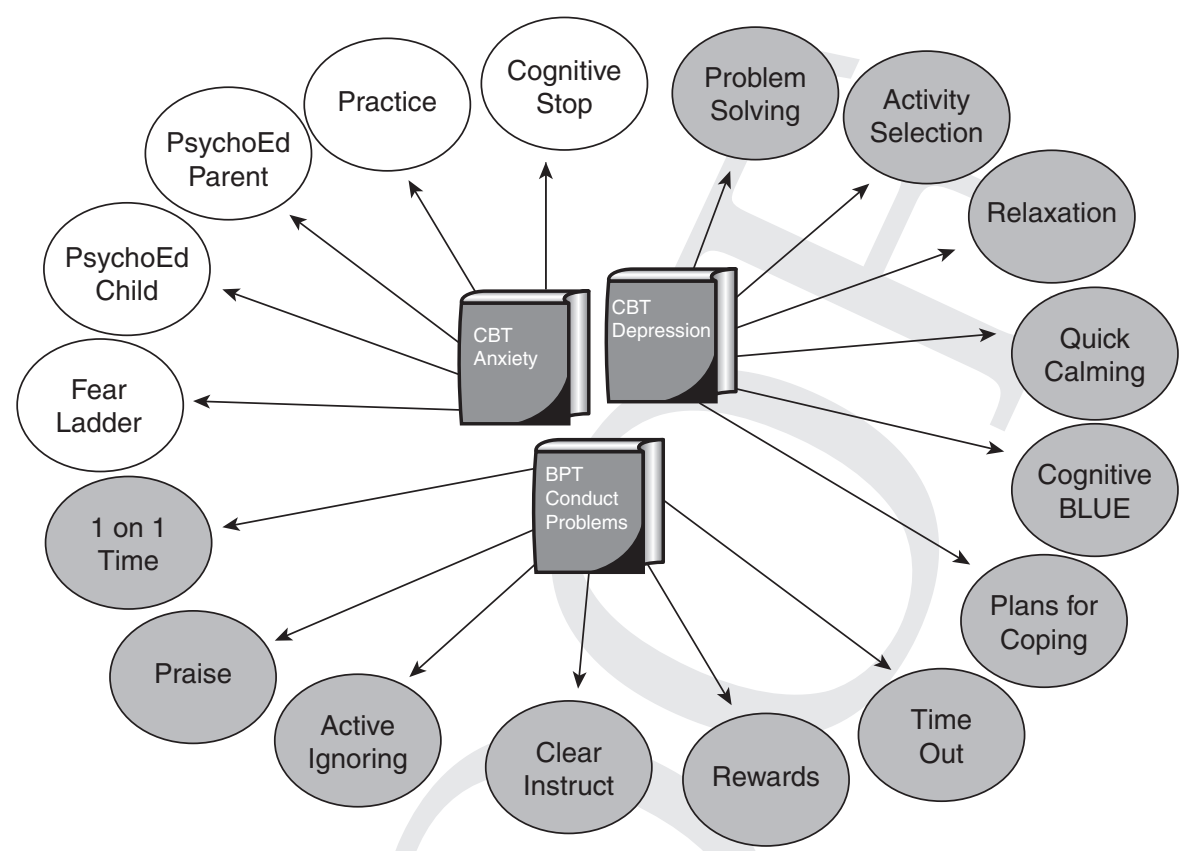

Figure 15.2. Design of Modular Approach to Therapy for Children ... (Chorpita \& Weisz, 2009), showing examples of modules derived from three forms of evidence-based psychotherapy - CBT for depression, CBT for anxiety, and behavioral parent training (BPT) for conduct-related problems and disorders. [Published with permission, John R. Weisz]

Modular Approach to Therapy for Children ... (MATCH; Chorpita \& Weisz, 2009).

MATCH is composed of multiple modules; each module is a 2-4-page description of one particular skill that youths and/or their caregivers need in order to overcome a particular problem. For example, using the problemsolving module, youths learn the STEPS procedure described earlier. In another module, caregivers learn to help their children improve behavioralregulation by targeting desirable positive behavior and not attending to undesirable but harmless behavior (e.g., whining). Some of the modules that were integrated to form MATCH are shown in Figure 15.2. Taken together, the full set of MATCH modules appears to target all three forms of self-regulation noted previously in this chapter - regulation of cognition (e.g., problem solving), emotion (e.g., relaxation, to reduce the intensity of anxiety), and behavior (e.g., praise). Some of the modules appear to encompass multiple forms of self-regulation. 
Examples of the hypothesized relation between modules and corresponding forms of self-regulation are shown in Table 15.2. For example, in the "activity selection" module for depression treatment, the young person identifies activities that reliably boost his or her mood and then schedules and performs those activities; this appears to be a means of supporting emotion regulation. In the "cognitive BLUE" module, the youth identifies cognitive errors related to depression, then learns to restructure the cognitions to make them more realistic; this appears to support cognitive regulation. And in the "practicing" module, the youth practices graduated exposure, as described previously; this module appears to support emotion regulation together with behavioral regulation. Collectively, the MATCH protocol could be seen as supporting a youth self-regulation agenda, with relevant skill learning supported by therapists, caregivers, and in some cases teachers (see Table 15.2).

The MATCH treatment manual includes a subset of modules designed mainly for depression treatment, another subset mainly for anxiety, and a third designed mainly for conduct problems, but while particular modules may "live" within one of those sections, most can be used for problems of other kinds (see Weisz \& Chorpita, 2011). For example, the module for problem solving "lives" in the depression section of the protocol, but it is often used in treatment of anxiety and conduct problems. The flexibility of MATCH includes the freedom to use only those modules that are needed for a particular youth, and the freedom from a fixed order in which modules or sessions must always occur. That freedom and flexibility, however, do not equate to "anything goes." Instead, treatment is guided by a series of flowcharts to inform decision making. In addition, decisions throughout episodes of care are guided by a system for weekly assessment of youth treatment response. We administer a standardized measure of youth internalizing and externalizing problems (Chorpita et al., 2010) and an idiographic measure of progress on the specific "top problems" youths and caregivers identified as most important at the beginning of treatment (Weisz et al., 2011); examples of these top problems include "gets into fights with his sister," "refuses to go to school," and "worries too much about things she can't control." These measures are administered weekly, and a webbased display makes the resulting information on youth treatment response available throughout treatment, for use in ongoing decisions such as which modules to use when, and whether to shift treatment focus.

This combination of two kinds of measures makes good sense, we believe, both for ongoing monitoring and feedback during treatment and for assessment of outcomes at the end of a treatment trial. The standardized 
Table 15.2. Selected Modules of the MATCH Protocol, and Hypothesized Self-Regulation Processes

\begin{tabular}{|c|c|c|}
\hline $\begin{array}{l}\text { Module } \\
\text { Name }\end{array}$ & Objective and Procedures & $\begin{array}{l}\text { Hypothesized Self-Regulation } \\
\text { Process }\end{array}$ \\
\hline $\begin{array}{l}\text { Active } \\
\text { Ignoring }\end{array}$ & $\begin{array}{l}\text { Caregiver learns to withdraw } \\
\text { attention from unwanted behaviors } \\
\text { and attend to desired behaviors }\end{array}$ & $\begin{array}{l}\text { Behavioral self-regulation } \\
\text { supported by change in caregiver } \\
\text { behavior }\end{array}$ \\
\hline $\begin{array}{l}\text { Activity } \\
\text { Selection }\end{array}$ & $\begin{array}{l}\text { The youth learns to identify } \\
\text { mood-boosting activities, then } \\
\text { schedule and practice them }\end{array}$ & $\begin{array}{l}\text { Emotional self-regulation } \\
\text { supported by caregiver behavior }\end{array}$ \\
\hline $\begin{array}{l}\text { Cognitive } \\
\text { BLUE }\end{array}$ & $\begin{array}{l}\text { The youth identifies cognitive errors } \\
\text { related to depression, then learns to } \\
\text { restructure the cognitions to make } \\
\text { them more realistic }\end{array}$ & $\begin{array}{l}\text { Cognitive self-regulation in the } \\
\text { service of emotion regulation }\end{array}$ \\
\hline $\begin{array}{l}\text { Cognitive } \\
\text { STOP }\end{array}$ & $\begin{array}{l}\text { The youth identifies cognitive errors } \\
\text { related to anxiety, then learns to } \\
\text { restructure the cognitions to make } \\
\text { them more realistic }\end{array}$ & $\begin{array}{l}\text { Cognitive self-regulation in the } \\
\text { service of emotion regulation }\end{array}$ \\
\hline $\begin{array}{l}\text { Daily Report } \\
\text { Card }\end{array}$ & $\begin{array}{l}\text { Teachers monitor youths' school } \\
\text { behavior, send daily report to } \\
\text { caregivers, who provide } \\
\text { consequences (e.g., privileges) to } \\
\text { youth at home }\end{array}$ & $\begin{array}{l}\text { Behavioral self-regulation } \\
\text { supported by teacher and } \\
\text { caregiver monitoring }\end{array}$ \\
\hline Fear Ladder & $\begin{array}{l}\text { The youth develops a list of feared } \\
\text { items, organized from most- to } \\
\text { least-feared, to prepare for practice } \\
\text { (exposure) }\end{array}$ & $\begin{array}{l}\text { Cognitive self-regulation in the } \\
\text { service of behavior regulation } \\
\text { and emotion regulation }\end{array}$ \\
\hline $\begin{array}{l}\text { Giving } \\
\text { Effective } \\
\text { Instructions }\end{array}$ & $\begin{array}{l}\text { Caregivers learn to give brief, clear } \\
\text { instructions so as to maximize youth } \\
\text { comprehension and compliance }\end{array}$ & $\begin{array}{l}\text { Behavioral and cognitive } \\
\text { self-regulation supported by } \\
\text { caregiver behavior }\end{array}$ \\
\hline $\begin{array}{l}\text { Learning to } \\
\text { Relax }\end{array}$ & $\begin{array}{l}\text { Youth learns progressive muscle } \\
\text { relaxation, guided imagery, } \\
\text { diaphragmatic breathing, to support } \\
\text { self-calming }\end{array}$ & $\begin{array}{l}\text { Behavioral self-regulation in the } \\
\text { service of emotion regulation }\end{array}$ \\
\hline Practicing & $\begin{array}{l}\text { Youth practices tolerating feared } \\
\text { stimuli, gradually mastering } \\
\text { increasingly fear-provoking items } \\
\text { via exposure/habituation }\end{array}$ & $\begin{array}{l}\text { Behavioral self-regulation in the } \\
\text { service of emotion regulation }\end{array}$ \\
\hline $\begin{array}{l}\text { Presenting a } \\
\text { Positive Self }\end{array}$ & $\begin{array}{l}\text { Youth learns and practices social } \\
\text { skills related to self-presentation }\end{array}$ & Behavioral self-regulation \\
\hline $\begin{array}{l}\text { Problem } \\
\text { Solving }\end{array}$ & $\begin{array}{l}\text { Youth learns and practices } \\
\text { systematic procedures for solving } \\
\text { personal problems }\end{array}$ & Behavioral self-regulation \\
\hline
\end{tabular}

(Published with Permission, John R. Weisz) 
measures of internalizing and externalizing problems make it possible to (a) link treatment response to prior research findings on these two broadband problem categories, (b) rely on the strength of standardized psychometrically sound measurement, (c) encompass the two broad problem categories encompassed by MATCH (i.e., internalizing includes anxiety and depression; externalizing includes conduct problems), and (d) employ a common array of content across all treated youths, so that their scores can be directly compared. The top problems measure is a useful complement to the standardized measurement. It provides an idiographic, psychometrically sound (Weisz et al., 2011) index of treatment response in relation to the specific concerns that matter most to the youths and their caregivers; for these consumers of mental health care, standardized internalizing and externalizing scores may matter less than the specific concerns that led them to seek treatment in the first place and that they identified at the outset as needing attention in treatment. Finally this combination of standardized and idiographic measures has the advantage of brevity - together the measures entail a total of only 15 questions, requiring just a minute or two of youth and caregiver time. We believe this helps maximize ongoing weekly participation by youths and caregivers, who might otherwise find the frequent assessment too burdensome; we needed to ensure that participants find the assessment acceptable enough that they continue to provide the data each week, thus informing clinician decision making during treatment and outcome evaluation at the end (as illustrated in the study described next).

\section{Testing the Redesigned Treatment: Randomized Effectiveness Trial of MATCH}

In structuring a test of this redesigned treatment approach, we tried to address the concerns noted earlier about unrepresentativeness of the youth psychotherapy evidence base. To test MATCH in real-world contexts, we based the test in everyday youth mental health service settings and in rather different geographic locations; our study took place in outpatient service settings in Massachusetts and Hawaii. We focused not on recruited youths but on youths who were referred to the outpatient settings through normal community channels. Treatment was provided not by research employees but by practicing clinicians. To address concerns about restrictive study inclusion criteria, we applied few exclusion rules, accepting youths who presented with problems and disorders involving anxiety, depression, misconduct, or any combination of these; most youths met criteria for multiple disorders. To address concerns about ethnic and racial homogeneity in 
many study samples, we sought and achieved an ethnically diverse sample. Finally, we identified two useful treatment conditions to compare to MATCH. Given the importance of testing new treatments against current practice, we included a usual care group. In addition, because MATCH was designed as an alternative to conventional EBPs, we included a third study condition: standard single-disorder treatment protocols. Therapists randomized to this condition were trained and supervised in the use of three separate protocols that mapped nicely onto the skills included in $\mathrm{MATCH}$ : Coping Cat (Kendall \& Hedtke, 2006), a CBT protocol for anxiety; Primary and Secondary Control Enhancement Training (PASCET; Weisz, Weersing, Valeri, \& McCarty, 1999), a CBT protocol for depression; and Defiant Children (Barkley, 1997), a behavioral parent training manual for conduct problems.

The study (Weisz et al., 2012) included 174 clinically referred youths, aged $7-13,70 \%$ boys, and ethnically diverse, with only $45 \%$ white. Standardized diagnostic assessment showed a broad array of diagnoses in the full sample, and the mean number of diagnoses per youth was 2.74. Treatment was carried out by 84 community clinicians who were employed in the ten sites; $80 \%$ were women, $56 \%$ were white, mean age was 40.6 , and most had been trained as social workers $(40 \%)$ or psychologists (24\%). Treatment was carried out in the ten outpatient sites, six in Massachusetts and four in Hawaii. The clinicians were randomly assigned to MATCH, standard protocol treatment, or usual care.

Most of our data analyses used a mixed effects regression approach that compared the groups on trajectories of change in the standardized measure (internalizing, externalizing, and total problems) and the idiographic "top problems" ratings. Results of these analyses are shown in Figure 15.3. The two bars at the left show the mean effect sizes found in the Weisz et al. $(2006,2013)$ meta-analyses of randomized trials pitting youth EBPs against usual care (described previously). The bars at the right show effect sizes for the primary outcome measures of the Weisz et al. (2012) RCT: standardized measures of internalizing and externalizing problems, and severity ratings for the individual top problems identified by youths and caregivers. As the figure shows, the effect sizes comparing standard treatment protocols to usual care were quite small; standard treatment did not outperform usual care. By contrast, the figure shows substantial effect sizes for all three outcome measures favoring MATCH over usual care, and also favoring MATCH over standard treatment protocols. In fact, the effect sizes showing MATCH outperforming usual care and standard protocols were noticeably larger than the mean effects found in the two EBP vs. usual care meta-analyses. 


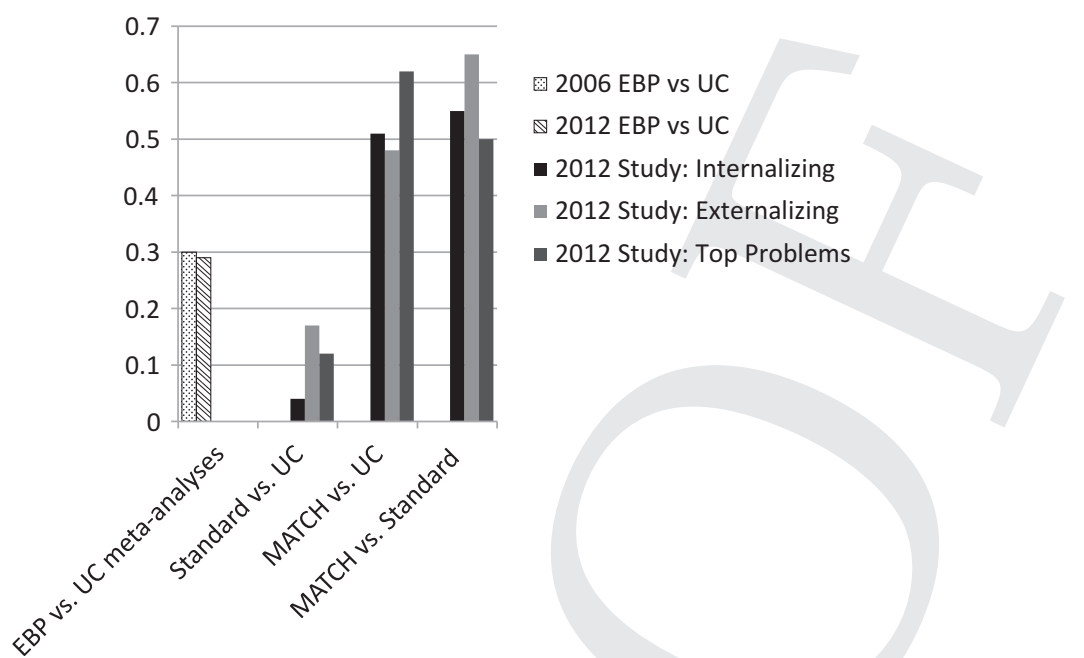

Figure 15.3. Effect size findings of the Weisz et al. (2012) randomized effectiveness trial comparing MATCH modular treatment to standard manual treatment and usual care. The two bars at the far left show the mean effect sizes found in two meta-analyses of randomized trials comparing evidence-based youth psychotherapies to usual care (Weisz et al., 2006, 2013). The other three sets of bars show effect size values on three primary outcome measures, in comparison of Standard Manuals to Usual Care, MATCH to Usual Care, and Match to Standard Manuals. [Published with permission, John R. Weisz]

In an additional analysis, we found that at the end of treatment youths receiving MATCH had significantly fewer diagnoses (using a standardized diagnostic interview) than youths receiving usual care. In sum, the findings (see Weisz et al., 2012) showed support for the effectiveness of MATCH on (a) widely studied dimensions of youth dysfunction - that is, internalizing and externalizing problems; (b) the specific problems identified by the consumers (i.e., youths and their caregivers) as most important to them; and (c) psychiatric diagnoses identified through standardized diagnostic interviews.

\section{Summary: Redesigning Youth Psychotherapy and Testing the Effects}

To address concerns about the appropriateness of many EBPs for real-world clinical practice, we designed MATCH, a protocol that integrates multiple components of EBPs for problems and disorders involving anxiety, depression, and misconduct. MATCH employs a modular design, with treatment decisions guided by clinical flowcharts and by a system for ongoing feedback on youth treatment response. A multisite randomized trial testing MATCH 
with a highly comorbid sample of clinically referred youths, treated in outpatient settings by clinical practitioners, showed that MATCH outperformed both usual care and standard single-disorder EBPs.

\section{Looking to the Future: Research on Intervention Design and Youth Self-Regulation}

The study described here is just one early step in exploring new approaches to integrative treatment design, building on the rich legacy of youth psychotherapy research. If the evidence in future studies continues to support this approach, we might imagine many iterations of integrative treatment synthesizing other EBP components, aimed at fitting well into clinical care contexts. When successful, this integrative approach - like the EBPs that produced its building blocks - promotes improved self-regulation in young people. We have focused here on three forms - cognitive, emotional, and behavioral self-regulation - that seem central to the EBPs, but more fully elaborated models may recognize other processes. A limitation of the treatment literature is that it focuses mainly on treatment procedures and the surface skills they address, with little discussion of underlying self-regulation processes. A challenge for future research will be delineating the linkage between youth psychotherapy and self-regulation, and boosting the impact of therapy on the most critical self-regulation processes. These goals may be pursued in at least three ways.

\section{Improving Assessment of Self-Regulation via Observable Behavior}

To understand the role of self-regulation processes in youth therapy, we will need enriched measurement models for valid assessment of the processes. A limitation of outcome measurement to date has been that much of the relevant measurement has relied on self-report and caregiver report, including standardized diagnostic interviews, standardized problem checklists, and symptom-specific measures. A similar limitation applies to the assessment of cognitive, emotional, and behavioral self-regulation processes; to the extent that these processes have been included in the measurement models of youth treatment research, the measures have mainly relied on youth and caregiver reports of the youths' thoughts, emotions, and behavior. Such verbal reports may miss the mark for a variety of reasons, including biased perspectives, incomplete opportunities to observe behavior across settings, and limited self-awareness (e.g., young people may not be able to gauge their own self-control processes accurately). Moreover, it is very difficult to 
keep participating youths and their caregivers blind to treatment condition, so the outcome data they provide can be difficult to interpret.

These problems can be addressed in part by multiplying the number of informants included. Teachers, for example, may be kept naïve to treatment condition and may have an informed perspective on youths during and after intervention in a treatment trial. On the other hand, neither teachers nor other non-caregivers are likely to have the rich observational base available to caregivers and to youths themselves. Moreover the reports of informants outside the family tend to show very modest levels of agreement with the reports of youths and their caregivers (Achenbach, McConaughy, \& Howell, 1987), raising questions about validity and proper interpretation of findings. Assessment of both self-regulation and outcome in treatment studies would be greatly enriched by a proliferation of measures based on direct observation. Direct observational methods are particularly feasible for behavioral outcomes and behavioral self-regulation - for example, reduced aggression in conduct treatment. However, observational methods are more elusive in the case of processes and outcomes that are hidden beneath the skin - for example, those involving cognitive and emotional self-regulation. This limitation suggests that there may be value in exploring measurement approaches derived from neuroscience, to which we now turn.

\section{Improving Assessment of Self-Regulation Processes under the Skin}

The neuroscience revolution has enriched our potential for understanding psychotherapy and self-regulation in ways that would have seemed sheer fantasy two decades ago. A limitation is that most of what we know thus far about the neural substrates of self-regulation comes from research with adults, including neuropsychological case studies (e.g., in which individuals suffer damage to specific brain structures) and neuroimaging and electrophysiological studies (Heatherton, 2011). However, the adult findings may inform efforts to measure and monitor self-regulation in relation to youth psychotherapy. The adult research tells us, for example, that the prefrontal cortex (PFC) plays a key role in the executive functions that are heavily involved in multiple forms of self-regulation (Curtis \& D'Esposito, 2003; Goldberg, 2001; Miller \& Cohen, 2001), with three regions of the PFC often noted as particularly critical. The ventromedial PFC (vMPFC) seems especially important to our ability to regulate appetitive, affective, and social behavior (Wagner \& Heatherton, 2010). Individuals suffering damage to this region of the brain have difficulty using social norms and feedback from others to make appropriate choices in social situations, and they tend 
to show disinhibited and inappropriate social behavior toward others (Beer, Heerey, Keltner, Scabini, \& Knight, 2003; Beer, John, Scabini, \& Knight, 2006). This tends to occur even when the individuals are fully aware that their behavior is socially inappropriate; this suggests that the vMPFC deficit is linked not to a failure to understand norms, but instead to a deficit in the ability to use cognitive control to overrule primary drives. By contrast, individuals who have lateral PFC damage are able to understand and inhibit inappropriate behaviors, but they find it difficult to plan and implement complex behavior, particularly when it involves pursuit of multiple goals. The tasks that are difficult for these individuals include those as simple as following a shopping list (Barcelo \& Knight, 2002) and as complex as completing the tasks needed to finish school. An additional frontal area that appears critical to self-regulation is the anterior cingulate cortex (ACC). Evidently, the ACC plays a key role in signaling the need to mobilize cognitive control to support self-regulation efforts, as is needed, for example, to overcome temptation and resist an inappropriate impulse (e.g., Kerns et al., 2004).

Self-Regulation of Cognitions. Therapeutic change often includes improved cognitive self-regulation, in such forms as suppressing or modifying thoughts that make certain situations unduly frightening, certain events unduly depressing, or ambiguous behavior by a peer seem more hostile than is actually the case. Findings (e.g., Mitchell et al., 2007) have suggested that the ACC monitors for conflict (e.g., between intrusion by unwanted thoughts and efforts to suppress those thoughts) and signals the need for additional cognitive control, and that the lateral PFC is active in efforts to implement and maintain thought suppression. Such findings bearing on thought suppression are intriguing and potentially relevant to therapeutic change. The focus on suppression could be nicely complemented in future research by a focus on modification of dysfunctional thoughts, which is especially relevant to the treatment of anxiety, depression, and conductrelated problems in youth.

Self-Regulation of Emotions. Problems and disorders involving anxiety, mood (e.g., depression), and aggressive and disruptive behavior are often linked to deficits in emotion regulation, a process that is being illuminated by extensive neuroscience research (e.g., Ochsner \& Gross, 2005), particularly within the subfield that has come to be called social cognitive neuroscience (Ochsner \& Lieberman, 2001). Much of this research points to the apparently central role of communication between the amygdala 
(a kind of emotion center in the limbic region of the brain) and the PFC. Evidence suggests that emotion regulation is linked to top-down regulation of amygdala activation by either the vMPFC (Johnstone, van Reekum, Urry, Kalin, \& Davidson, 2007), the lateral PFC (Ochsner, Bunge, Gross, \& Gabrieli, 2002), or possibly the vMPFC mediating the impact of the lateral PFC on the amygdala. Findings over the past decade point to an apparent breakdown in functioning of the amygdala-PFC circuit in cases of adult major depressive disorder (Johnstone et al., 2007), PTSD (Shin et al., 2005), and borderline personality disorder (Donegan et al., 2003). In the future, it may be valuable to extend this work to youth populations and to assess, for example, whether neuroimaging focused on the amygdalaPFC circuit can enrich our understanding of what processes must be in place in the maturing brain for therapeutic change in emotion regulation to occur.

Self-Regulation of Behavior. Behavioral regulation is, in some respects, the holy grail of psychotherapy. Managing or modifying thoughts and emotions, while important, may be seen by many as valuable largely because they are steps needed to achieve behavioral control - for example, over avoidance (in cases of anxiety), vegetative behavior and self-harm (in cases of depression), aggression and criminal behavior (in conduct-related problems and disorders), and of course substance abuse and dependence in related disorders. Much of the neuroscience related to behavioral control has focused on the role of the brain's reward system, in particular the mesolimbic dopamine system, through which certain behaviors activate dopamine neurons located in the nucleus accumbens (NAcc). NAcc activation is associated with a variety of rewarding events (Cloutier, Heatherton, Whalen, \& Kelley, 2008), ranging from eating (O'Doherty, Dyan, Friston, Critchley, \& Dolan, 2003) to drug use (Zubieta et al., 2005). Evidence suggests that regulating behaviors associated with NAcc activation - that is, showing self-control - requires top-down control from the PFC, with findings pointing to roles for the lateral PFC and the ACC. In general, the research suggests that failures in behavioral regulation are often due to overactivation of the NAcc, which resists the regulating influence of the PFC, with a resulting loss of selfcontrol (Heatherton, 2011). Much of the relevant NAcc-PFC research has focused on adult habit-related and addictive behaviors, including overeating and substance abuse. In the future, it may be valuable to extend this work to younger populations and assess the extent to which the findings generalize to psychotherapy process and outcome in relation to the most common youth internalizing and externalizing conditions. 


\section{Understanding the Most Effective and Efficient Ways to Promote Self-Regulation}

Along with improved measurement, we will need to identify the most effective methods for promoting self-regulation, and find ways to build these methods into youth psychotherapies. The evidence reviewed earlier in this chapter suggests that treatment developers have generated some reasonably effective strategies. However, the modest magnitude of the effect sizes indicates that there is considerable room for improvement. We may find clues to more potent strategies in social, developmental, and cognitive psychology research. In one illustrative body of work, Gollwitzer, Oettingen and colleagues (described in this volume) have studied processes associated with the pursuit of goals, many of which involve critical forms of selfregulation (e.g., Gollwitzer \& Oettingen, 2011; Oettingen, 2012; Oettingen \& Gollwitzer, 2010). Their work distinguishes between goal intentions (i.e., an individual's intent to attain a particular objective - e.g., eat low-fat food or enter an unfamiliar social situation) and implementation intentions (i.e., the intention to carry out a specific plan regarding what to do in a particular situation; Gollwitzer, 1999). Implementation intentions serve goal intentions by clarifying what will be done to attain the goal, and when, where, and how it will be done. One focus of work described recently by Oettingen (2012) is an effort to identify specific strategies people can use to regulate their own autonomous pursuit of goals. One promising strategy, derived from fantasy realization theory (Oettingen, 1999; 2012), involves mentally contrasting the future and reality, a meta-cognitive strategy that has been linked to enhanced goal pursuit when expectations of success are high (and diminished goal pursuit when expectations are low). Incorporating and adapting strategies like these, and others derived from research in social-cognitive psychology and allied specialties, could help boost the impact of youth psychotherapies on critical self-regulation processes.

\section{Conclusion}

In this chapter I have argued that improving self-regulation is a central objective underlying much of youth psychotherapy. Indeed, three forms of self-regulation - cognitive, emotional, and behavioral - may be viable candidates in the search for therapeutic "mechanisms of change." The search for the most effective evidence-based psychotherapies (EBPs) continues, and innovations such as the integrative MATCH protocol may provide useful advances in that search. As a complement to such advances, efforts to build the most potent treatments may be accelerated by a focus on 
measuring, monitoring, and promoting self-regulation in the context of youth psychotherapy.

\section{REFERENCES}

Achenbach, T. M., McConaughy, S. H., \& Howell, C. T. (1987). Child/adolescent behavioral and emotional problems: Implications of cross-informant correlations for situational specificity. Psychological Bulletin, 101, 213-232.

Addis, M. E., \& Krasnow, A. D. (2000). A national survey of practicing psychologists' attitudes toward psychotherapy treatment manuals. Journal of Consulting and Clinical Psychology, 68, 331-339.

Angold, A., Costello, E. J., \& Erkanli, A. (1999). Comorbidity. Journal of Child Psychology and Psychiatry, 40, 57-87.

Barcelo, F., \& Knight, R. T. (2002). Both random and perseverative errors underlie WCST deficits in prefrontal patients. Neuropsychologia, 40, 349-356.

Barkley, R. (1997). Defiant children (2nd ed.). New York: Guilford.

Beer, J. S., Heerey, E. A., Keltner, D., Scabini, D., \& Knight, R. T. (2003). The regulatory function of self-conscious emotion: Insights from patients with orbitofrontal damage. Journal of Personality and Social Psychology, 85, 594-604.

Beer, J. S., John, O. P., Scabini, D., \& Knight, R. T. (2006). Obitofrontal cortex and social behavior: Integrating self-monitoring and emotion-cognition interactions. Journal of Cognitive Neuroscience, 18, 871-880.

Casey, R. J., \& Berman, J. S. (1985). The outcome of psychotherapy with children. Psychological Bulletin, 98, 388-400.

Chorpita, B. F., \& Daleiden, E. L. (2009). Mapping evidence-based treatments for children and adolescents: Application of the distillation and matching model to 615 treatments from 322 randomized trials. Journal of Consulting and Clinical Psychology, 77, 566-579.

Chorpita, B. F., Daleiden, E. L., \& Weisz, J. R. (2005). Identifying and selecting the common elements of evidence based interventions: A distillation and matching model. Mental Health Services Research, 7, 5-20.

Chorpita, B. F., Reise, S., Weisz, J. R., Grubbs, K., Becker, K. D., Krull, J. L., \& the Research Network on Youth Mental Health. (2010). Evaluation of the Brief Problem Checklist: Child and caregiver interviews to measure clinical progress. Journal of Consulting and Clinical Psychology, 78, 526-536.

Chorpita, B. F., \& Weisz, J. R. (2009). Modular approach to therapy for children with anxiety, depression, trauma, or conduct problems (MATCH-ADC). Satellite Beach, FL: PracticeWise, LLC.

Cloutier, J., Heatherton, T. F., Whalen, P. J., \& Kelley, W. M. (2008). Are attractive people rewarding? Sex differences in the neural substrates of facial attractiveness. Journal of Cognitive Neuroscience, 20, 941-951.

Cohen, J. (1988). Statistical power analysis for the behavioral sciences (2nd ed.). Hillsdale, NJ: Lawrence Erlbaum Associates.

Curtis, C. E., \& D'Esposito, M. (2003). Success and failure suppressing reflexive behavior. Journal of Cognitive Neuroscience, 15, 409-418.

Donegan, N. H., Sanislow, C. A., Blumberg, H. P., Fullbright, R. K., Lacadie, C., et al. (2003). Amygdala hyperreactivity in borderline personality disorder: Implications for emotional dysregulation. Biological Psychiatry, 54, 1284-1293. 
Goldberg, E. (2001). The executive brain: The frontal lobes and the civilized mind. New York: Oxford University Press.

Gollwitzer, P. M. (1999). Implementation intentions: Strong effects of simple plans. American Psychologist, 54, 493-503.

Gollwitzer, P. M., \& Oettingen, G. (2011). Planning promotes goal striving. In K. D. Vohs \& R. F. Baumeister (Eds.), Handbook of self-regulation: Research, theory, and applications (2nd ed., pp. 162-185). New York: Guilford.

Hammen, C., Rudolph, K., Weisz, J. R., Burge, D., \& Rao, U. (1999). The context of depression in clinic-referred youth: Neglected areas in treatment. Journal of the American Academy of Child and Adolescent Psychiatry, 38, 64-71.

Havik, O. E., \& Van den Bos, G. R. (1996). Limitations of manualized psychotherapy for everyday clinical practice. Clinical Psychology: Science and Practice, 3, 264-267.

Heatherton, T. F. (2011). Neuroscience of self and self-regulation. Annual Review of Psychology, 62, 363-390.

Jensen, A. L., \& Weisz, J. R. (2002). Assessing match and mismatch between practitionergenerated and standardized interview-generated diagnoses for clinic-referred children and adolescents. Journal of Consulting and Clinical Psychology, 70, 158-168.

Johnstone, T., van Reekum, C. M., Urry, H. L., Kalin, N. H., \& Davidson, R. J. (2007). Failure to regulate: Counterproductive recruitment of top-down prefrontal-subcortical circuitry in major depression. Journal of Neuroscience, 27, 8877-8884.

Kazdin, A. E., Bass, D., Ayers, W. A., \& Rodgers, A. (1990). Empirical and clinical focus of child and adolescent psychotherapy research. Journal of Consulting \& Clinical Psychology, 58, 729-740.

Kendall, P. C., \& Hedtke, K. (2006). Cognitive-behavioral therapy for anxious children: Therapist manual (3rd ed.). Ardmore, PA: Workbook. Retrieved from http:// workbookpublishing.com

Kerns, J. G., Cohen, J. D., MacDonald, A. W., Cho, R. Y., Stenger, V. A., \& Carter, C. S. (2004). Anterior cingulate conflict monitoring and adjustments in control. Science, 303, 1023-1026.

Levitt, E. E. (1957). The results of psychotherapy with children: An evaluation. Journal of Consulting Psychology, 21, 189-196.

Levitt, E. E. (1963). Psychotherapy with children: A further evaluation. Behaviour Research and Therapy, 60, 326-329.

McGraw, K. O., \& Wong S. P. (1992). A common language effect size statistic. Psychological Bulletin, 111, 361-365.

Miller, E. K., \& Cohen, J. D. (2001). An integrative theory of prefrontal cortex function. Annual Review of Neuroscience, 24, 167-202.

Mitchell, J. P., Heatherton, T. F., Kelley, W. M., Wyland, C. L., Wegner, D. M., \& McCrae, C. N. (2007). Separating sustained from transient aspects of cognitive control during thought suppression. Psychological Science, 18, 292-297.

Ochsner, K. N., Bunge, S. A., Gross, J. J., \& Gabrieli, J. D. (2002). Rethinking feelings: An FMRI study of the cognitive regulation of emotion. Journal of Cognitive Neuroscience, $14,1215-1229$.

Ochsner, K. N., \& Gross, J. J. (2005). The cognitive control of emotion. Trends in Cognitive Science, 9, 242-249.

Ochsner, K. N., \& Lieberman, M. D. (2001). The emergence of social cognitive neuroscience. American Psychologist, 56, 717-734. 
O’Doherty, J. P., Dyan, P., Friston, K., Critchley, H., \& Dolan, R. J. (2003). Temporal different models and reward-related learning in the human brain. Neuron, 38, 329337.

Oettingen, G. (1999). Free fantasies about the future and the emergence of developmental goals. In J. Brandtstädter \& R. M. Lerner (Eds.), Action \& self-development: Theory and research through the life span (pp. 315-342). Thousand Oaks, CA: Sage.

Oettingen, G. (2012). Future thought and behavior change. European Review of Social Psychology, 23, 1-63.

Oettingen, G., \& Gollwitzer, P. M. (2010). Strategies of setting and implementing goals: Mental contrasting and implementation intentions. In J. E. Maddux \& J. P. Tangney (Eds.), Social psychological foundations of clinical psychology (pp. 114-135). New York: Guilford.

Schoenwald, S. K., Kelleher, K., Weisz, J. R., \& the Research Network on Youth Mental Health. (2008). Building bridges to evidence-based practice: The MacArthur Foundation Child System and Treatment Enhancement Projects (Child STEPs). Administration and Policy in Mental Health and Mental Health Services Research, 35, 66-72.

Shapiro, D. A., \& Shapiro, D. (1982). Meta-analysis of comparative therapy outcome studies: A replication and refinement. Psychological Bulletin, 92, 581-604.

Shin, L. M., Wright, C. I., Cannistraro, P. A., Wedig, M. M., McMullin, K. A., et al. (2005). An fMRI study of amygdala and medial prefrontal cortex responses to overtly presented fearful faces in posttraumatic stress disorder. Archives of General Psychiatry, $62,273-281$.

Silverman, W. K., \& Hinshaw, S. P. (2008). The second special issue on evidence-based psychosocial treatments for children and adolescents: A 10-year update. Journal of Child and Adolescent Clinical Psychology, 37, 1-7.

Smith, M. L., \& Glass, G. V. (1977). Meta-analysis of psychotherapy outcome studies. American Psychologist, 32, 752-760.

Southam-Gerow, M. A., Weisz, J. R., Chu, B. C., McLeod, B. D., Gordis, E. B., \& ConnorSmith, J. K. (2010). Does CBT for youth anxiety outperform usual care in community clinics? An initial effectiveness test. Journal of the American Academy of Child of Adolescent Psychiatry, 49, 1043-1052.

Southam-Gerow, M. A., Weisz, J. R., \& Kendall, P. C. (2003). Youth with anxiety disorders in research and service clinics: Examining client differences and similarities. Journal of Clinical Child and Adolescent Psychology, 32, 375-385.

Wagner, D. D., \& Heatherton, T. F. (2010). Giving in to temptation: The emerging cognitive neuroscience of self-regulatory failure. In R. F. Baumeister \& K. D. Vohs (Eds.), The handbook of self-regulation (2nd ed., pp. 41-63). New York: Guilford.

Weisz, J. R. (2004). Psychotherapy for children and adolescents: Evidence-based treatments and case examples. Cambridge: Cambridge University Press.

Weisz, J. R., \& Addis, M. E. (2006). The research-practice tango and other choreographic challenges: Using and testing evidence-based psychotherapies in clinical care settings. In C. D. Goodheart, A. E. Kazdin, \& R. J. Sternberg (Eds.), Evidence-based psychotherapy: Where practice and research meet (pp. 179-206). Washington, DC: American Psychological Association.

Weisz, J. R., \& Chorpita, B. F. (2011). Mod squad for youth psychotherapy: Restructuring evidence-based treatment for clinical practice. In P. C. Kendall (Ed.), Child and 
adolescent therapy: Cognitive-behavioral procedures (4th ed., pp. 379-397). New York: Guilford Press.

Weisz, J. R., Chorpita, B. F., Frye, A., Ng, M. Y., Lau, N., et al. (2011). Youth top problems: Using idiographic, consumer-guided assessment to identify treatment needs and track change during psychotherapy. Journal of Consulting and Clinical Psychology, 79, 369380.

Weisz, J. R., Chorpita, B. F., Palinkas, L. A., Schoenwald, S. K., Miranda, J., et al. (2012). Testing standard and modular designs for psychotherapy with youth depression, anxiety, and conduct problems: A randomized effectiveness trial. Archives of General Psychiatry, 69, 274-282.

Weisz, J. R., \& Gray, J. S. (2008). Evidence-based psychotherapies for children and adolescents: Data from the present and a model for the future. Child and Adolescent Mental Health, 13, 54-65.

Weisz, J. R., Jensen-Doss, A. J., \& Hawley, K. M. (2005). Youth psychotherapy outcome research: A review and critique of the evidence base. Annual Review of Psychology, 56, 337-363.

Weisz, J. R., Jensen-Doss, A., \& Hawley, K. M. (2006). Evidence-based youth psychotherapies versus usual clinical care: A meta-analysis of direct comparisons. American Psychologist, 61, 671-689.

Weisz, J. R., Kuppens, S., Eckshtain, D., Ugueto, A. M., Hawley, K. M., \& Jensen-Doss, A. (2013). Performance of evidence-based youth psychotherapies compared with usual clinical care: A multilevel meta-analysis. JAMA Psychiatry, 70, 750-761.

Weisz, J. R., Ng, M. N., Rutt, C., Lau, N., \& Masland, S. A. (2013). Psychotherapy for children and adolescents. In M. J. Lambert (Ed.), Bergin and Garfield's handbook of psychotherapy and behavior change (6th ed., pp. 541-586). New York: John Wiley \& Sons.

Weisz, J. R., Southam-Gerow, M. A., Gordis, E. B., Connor-Smith, J. K., Chu, B. C., et al. (2009). Cognitive-behavioral therapy versus usual clinical care for youth depression: An initial test of transportability to community clinics and clinicians. Journal of Consulting and Clinical Psychology, 77, 383-396.

Weisz, J. R., Weersing, V. R., Valeri, S. M., \& McCarty, C. A. (1999). Therapist's Manual PASCET: Primary and secondary control enhancement training program. Los Angeles: University of California Press.

Weisz, J. R., Weiss, B., Alicke, M. D., \& Klotz, M. L. (1987). Effectiveness of psychotherapy with children and adolescents: A meta-analysis for clinicians. Journal of Consulting \& Clinical Psychology, 55, 542-549.

Weisz, J. R., Weiss, B., Han, S. S., Granger, D. A., \& Morton, T. (1995). Effects of psychotherapy with children and adolescents revisited: A meta-analysis of treatment outcome studies. Psychological Bulletin, 117, 450-468.

Westen, D., Novotny, C. M., \& Thompson-Brenner, H. (2004). The empirical status of empirically supported therapies: Assumptions, findings, and reporting in controlled trials. Psychological Bulletin, 130, 631-663.

Zubieta, J. K., Heitzeg, M. M., Xu, Y., Koeppe, R. A., Ni, L., Guthrie, S., \& Domino, E. F. (2005). Regional cerebral blood flow responses to smoking in tobacco smokers after overnight abstinence. American Journal of Psychiatry, 162, 567-577. 